\title{
Teaching Mixed Strategy Nash Equilibrium to Undergraduates
}

Kenneth Garrett and Evan Moore

\begin{abstract}
The authors present a simple and effective method for improving student comprehension of mixed strategies. After reviewing available texts, and with some trial and error, we feel that this method eases the teaching of the information and increases the students' ability to understand and retain concepts. Through a simple alteration to the normal form of a game, students can clearly see the results of employing mixed strategies, practise calculating expected payoffs, visualise the accompanying 'indifference' in the payoffs, and recognise the mixed strategy Nash equilibrium in a familiar way.
\end{abstract}

\section{Introduction}

Understanding the concept of an optimal mixed strategy is not an easy task for the inexperienced undergraduate student. Anecdotal evidence suggests that the approaches used in a variety of textbooks to present this material leave students feeling that finding optimal mixed strategies and the resulting Nash equilibria is nothing more than a mathematical exercise. We propose a simple method that reveals how a mixed strategy Nash equilibrium (MSNE) is similar to Nash equilibrium in pure strategies. Additionally, the proposed method aids students in grasping the concept of 'indifference' for each player when faced with an optimal mixed strategy. Our approach is easy to implement with a simple modification to the normal form of a game, and we have found it to be quite effective in helping students to master the concept of mixed strategies.

Mixed strategies involve a player randomly choosing among pure strategies according to given probabilities. Bernheim and Whinston (2008) define a mixed strategy as a player using,'a rule to randomize over the choice of a strategy'. The 
definition given by Pindyck and Rubinfeld (2005) may be clearer, as they define a mixed strategy as a'strategy in which a player makes a random choice among two or more possible actions, based on a set of chosen probabilities'. The value of mixed strategies should not be underestimated. There are a number of motivations given in undergraduate textbooks regarding the need for, and use of, mixed strategies. Pindyck and Rubinfeld (2005) state that, 'there are games... in which a pure strategy is not the best way to play'. McCain (2004) describes the need for a mixed strategy when the player would benefit from being 'unpredictable, so that the opposition cannot guess which strategy is coming and prepare accordingly'. Bernheim and Whinston (2008) support this by motivating their discussion on mixed strategies with,'the key to success... is unpredictability.... The most obvious choice is to make choices randomly'. Baldani et al. (2005) write:'Not all games have pure strategy Nash equilibria in which each player chooses a single strategy with probability one. There are important economic applications that only have solutions in mixed strategies in which a player randomizes by choosing the probabilities for playing the possible pure strategies.'

There is a wide variety of instances of mixed strategies in the 'real world'. These include uses in games such as 'Rock-Paper-Scissors' to the world of sports. Examples in sports include baseball pitchers alternating between various pitches, such as a curveball, sinker and fastball, to keep hitters off-balance, or football offences varying between the passing and running attacks to keep defences 'honest'. Two articles in the American Economic Review look at mixed strategies in sports; Chiappori et al. (2002) investigate the use of mixed strategies during penalty kicks in professional soccer matches and Walker and Wooders (2001) review mixed strategies employed by servers to opponents' forehands or backhands in professional tennis matches. A nice resource for articles and movies that mention or involve mixed strategies, and game theory in general, is www.gametheory.net. It includes articles involving the concept of mixed strategies in dealing with terrorism, tax cheats and the Internal Revenue Service, playing poker, quantum mechanics, and how beating the S\&P is like a game of 'matching pennies.' 1 These articles are from a range of sources including magazines such as the Economist, Nature, Newsweek and Slate, to the news divisions of $A B C$ and $C B S$, and a variety of newspapers. There are also instances of mixed strategies in films. John Kay (1994) writes:'But the greatest paradox of all is that it is often best to adapt random behaviour in chicken games. It is possible to write down the mathematics of the problem faced by the two groups of boys in Stand By Me, and to show that in general a good solution for each is to swerve sometimes and to stick sometimes. You can even calculate how often you should swerve and how often you should stick.' Other examples of 'Chicken' can be found in the films Rebel without a Cause and Footloose. A game of the 'Battle of Wits' is in the The Princess Bride, a discussion of which is presented in Bernheim and Whinston 
(2008). Given the wide range of applications where the uses of mixed strategies apply in decision making, we feel an improved understanding of the topic is useful to undergraduate economics students.

\section{Textbook approaches}

We selected a variety of universities to see which textbooks were used in Intermediate Microeconomics, Managerial Economics and Mathematical Economics. We used courses from Massachusetts Institute of Technology, University of California at Berkeley, Auburn University, University of Georgia, Wake Forest University, University of New Mexico, Amherst College and University of Nebraska at Omaha. ${ }^{2}$ We believe that many other colleges and universities are likely to use these, or similar, textbooks in their courses. The goals of our review were to investigate: 1) the mathematical method for determining the optimal mixed strategies; and 2) how the end results are visually presented in the textbook.

The textbooks we reviewed fell into one of two categories regarding the methodology used to determine the optimal mixed strategies:

1 Determining the mixed strategies algebraically, generally by solving for the probability of one strategy, $p$, and the probability of the other strategy choice as a function of the first strategy, i.e. 1-p, and thus making the opponent indifferent (Hay et al.; Nicholson and Snyder; McGuigan et al.; Neilson and Winter; Pindyck and Rubinfeld; Varian).

2 Determining the mixed strategies by taking the derivative of a linear function that represents players' strategy expectations. This method, while not difficult, was the least user-friendly and it is easy to see that it gives the same results as the simpler algebraic method (Baldani et al., 2005).

We did not find any textbook approach that includes our proposed method for improving student learning and comprehension of the concept of mixed strategies and the resulting Nash equilibrium.

\section{In the classroom}

To get the students engaged in the concept of mixed strategies, we have them group into pairs and play a few rounds of a standard 'matching pennies' game. We provide them with very little explanation of mixed strategies other than broadly discussing making the other player indifferent between strategy choices and we do not show any technique to solve for an optimal mixed strategy at this point. Nevertheless, it typically does not take the students long to arrive at the optimal mixed strategy, i.e. probability weights of $1 / 2$ for each of the strategies. In some 
instances we followed this with the pairs playing a few rounds of 'Rock-PaperScissors' and the students quickly conclude that the optimal mixed strategy involves attaching a probability weight of one-third to each strategy. After playing these zero sum games, we introduce a simple $2 \times 2$ non-zero sum game, such as the one shown in Figure 1. For the purposes of this paper we show each player's best response to the other player's choice by underscoring the payoff. Note that this game does not have a Nash equilibrium in pure strategies, as evidenced by no outcome having both payoffs in a cell underscored.

We then ask the students to propose a few possible probability weights for equilibrium mixed strategies, reminding them that the aim of a player's mixed strategy is to make the other player indifferent between his strategy choices. Frequently they respond with weights of $1 / 2$ for each strategy. We then add an additional row for player 1 and column for player 2 to the original game, labelling each strategy to reflect the selected weights. This is shown in Figure 2. The expected payoffs in the new cells by row are calculated using the weights of player 2's mixed strategy. For example, consider the expected payoffs in the cell resulting from player 1 playing $A$ and player 2 playing the mixed strategy of $1 / 2 C$ and $1 / 2 D$. Player 1 's expected payoff from playing $A$ is $1 / 2^{*} 4+1 / 2^{*} 12=8$. Player 2 's expected payoff when player 1 plays $A$ is $1 / 2^{*} 25+1 / 2^{*} 5=15$. These expected payoffs are listed in the cell in the upper right corner of Figure 2. Similarly, the expected payoffs from player 2 playing a strategy versus player 1's mixed strategy are calculated using weighted values of the payoffs by column. ${ }^{3}$ At this point we once again identify each player's best response for this $3 \times 3$ matrix using underscores. We then point out to the students that there is no Nash equilibrium with the $1 / 2$ probability weights.

Once again we reintroduce the notion of indifference, and that a player is indifferent between strategy choices if the expected payoff is the same for that player. We note that this means player 1 will have the same expected payoff from playing $A, B$ or the optimal mixed strategy when facing the optimal mixed strategy

Figure 1: A 2x2 non-zero sum game

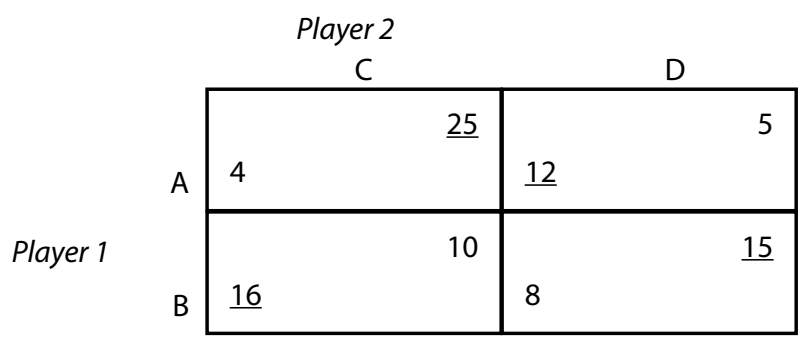


Figure 2: Including mixed strategies with probability weights of $1 / 2$

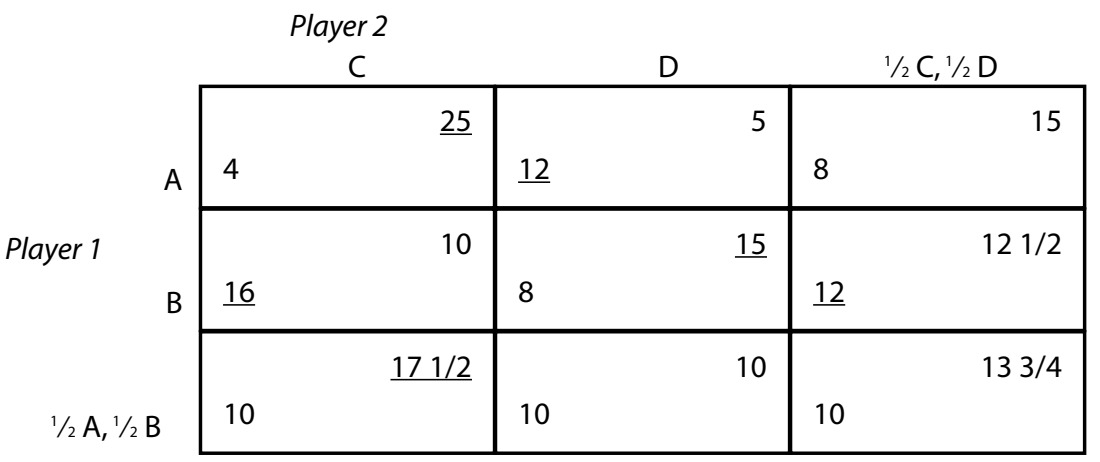

choice for player 2, i.e. all of player 1's expected payoffs will be the same in the optimal mixed strategy column for player 2. Likewise, we take the time to point out that player 2's expected payoffs will be the same when facing the optimal mixed strategy from player 1, i.e. all of the payoffs to player 2 are identical in the same mixed strategy row for player 1.

At this point in class we introduce how to solve for the optimal mixed strategy choices using the common textbook techniques. This is done by equating a player's expected values from each of her strategy choices, i.e. player 2 is mixing between strategies $C$ and $D$ to make player 1 indifferent between playing strategies $A$ and $B$, while player 1 is mixing between strategies $A$ and $B$ to make player 2 indifferent between strategies $C$ and D. We provide the solution for the game in Figure 1 using the standard algebraic approach. We use this approach because generally the students seem more comfortable using algebra and because six of the seven textbooks reviewed that cover mixed strategies use an algebraic approach. ${ }^{4}$ The solution using calculus is provided in the appendix.

Player 2 mixing to make Player 1 indifferent; Player 1 mixing to make Player 2 indifferent

$$
\begin{aligned}
\mathrm{EV}(\mathrm{A})=\mathrm{EV}(\mathrm{B}) & \\
4 \mathrm{P}_{2 \mathrm{C}}+12\left(1-\mathrm{P}_{2 \mathrm{C}}\right) & =16 \mathrm{P}_{2 \mathrm{C}}+8\left(1-\mathrm{P}_{2 \mathrm{C}}\right) \\
4 \mathrm{P}_{2 \mathrm{C}}+12-12 \mathrm{P}_{2 \mathrm{C}} & =16 \mathrm{P}_{2 \mathrm{C}}+8-8 \mathrm{P}_{2 \mathrm{C}} \\
12-8 \mathrm{P}_{2 \mathrm{C}} & =8 \mathrm{P}_{2 \mathrm{C}}+8 \\
4 & =16 \mathrm{P}_{2 \mathrm{C}} \\
\mathrm{P}_{2 \mathrm{C}} & =4 / 16=1 / 4 \\
\mathrm{P}_{2 \mathrm{D}} & =1-\mathrm{P}_{2 \mathrm{C}}=3 / 4
\end{aligned}
$$

$$
\begin{gathered}
\mathrm{EV}(\mathrm{C})=\mathrm{EV}(\mathrm{D}) \\
25 \mathrm{P}_{1 \mathrm{~A}}+10\left(1-\mathrm{P}_{1 \mathrm{~A}}\right)=5 \mathrm{P}_{1 \mathrm{~A}}+15\left(1-\mathrm{P}_{1 \mathrm{~A}}\right)
\end{gathered}
$$$$
25 \mathrm{P}_{1 \mathrm{~A}}+10-10 \mathrm{P}_{1 \mathrm{~A}}=5 \mathrm{P}_{1 \mathrm{~A}}+15-15 \mathrm{P}_{1 \mathrm{~A}}
$$$$
15 \mathrm{P}_{1 \mathrm{~A}}+10=15-10 \mathrm{P}_{1 \mathrm{~A}}
$$$$
25 \mathrm{P}_{1 \mathrm{~A}}=5
$$$$
\mathrm{P}_{1 \mathrm{~A}}=1 / 5
$$$$
\mathrm{P}_{1 \mathrm{~B}}=1-\mathrm{P} 1 \mathrm{~A}=4 / 5
$$ 
Figure 3: Including the optimal mixed strategies

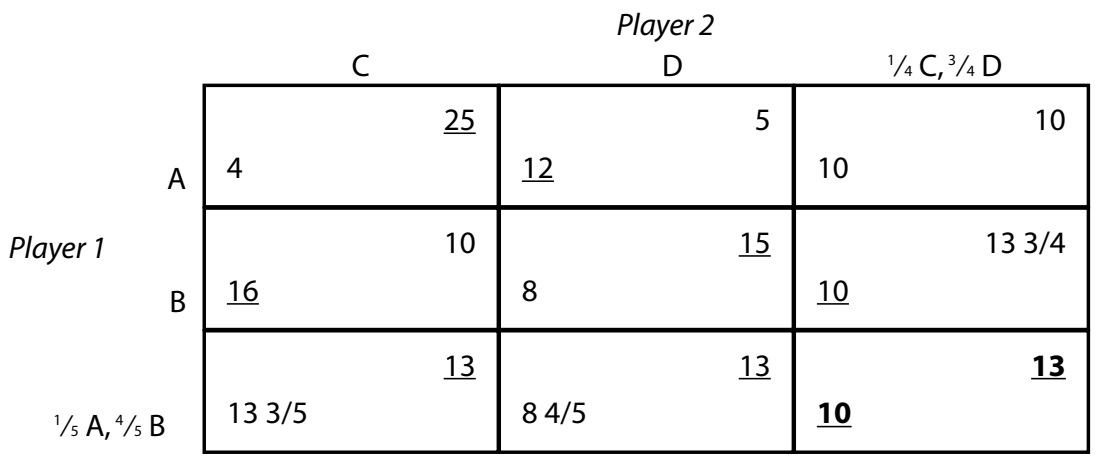

We follow this by adding the newly found optimal mixed strategies to Figure 1 to create Figure 3. We then calculate the new expected payoffs for each of the cells involving the mixed strategies. We stress to the students that, when properly weighted, the $3 \times 3$ game now clearly shows each player's indifference to the other players' mixed strategy choice.

Once again we underscore the best responses for each player. This results in the MSNE as shown in the bottom right cell of Figure 3. We find that this simple addition, including the mixed strategies in the normal form, enhances the ability of students to grasp the concept of indifference, calculate expected payoffs, and visualise the MSNE in a similar way to Nash equilibrium in pure strategies. ${ }^{5}$

\section{Conclusion}

We present a simple and effective method for improving student comprehension of mixed strategies. After reviewing available texts, and with some trial and error, we feel that this method eases the teaching of the information and increases the students' ability to understand and retain the concepts. Through a simple alteration to the normal form of a game, students can clearly see the results of employing mixed strategies, practise calculating expected payoffs, visualise the accompanying 'indifference' in the payoffs, and recognise the mixed strategy Nash equilibrium in a familiar way. 


\section{Appendix}

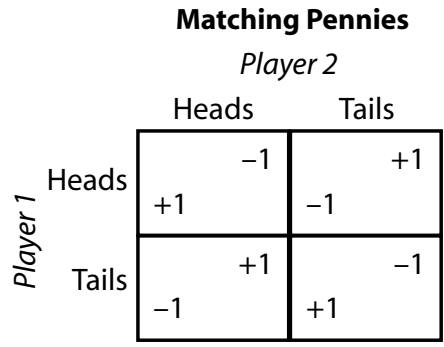

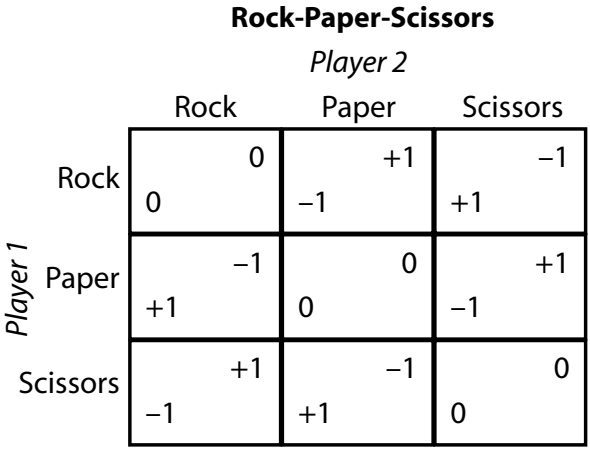

Solving for the optimal mixed strategies illustrated in Figure 3 using calculus.

$$
\begin{aligned}
& E\left(\Pi_{1}\right)=p q 4+p(1-q) 12+(1-p) q 16+(1-p)(1-q) 8 \\
& \frac{\partial E\left(\Pi_{1}\right)}{\partial p}=4 q+(1-q) 12-16 q-(1-q) 8=0 \\
& \frac{\partial E\left(\Pi_{1}\right)}{\partial p}=-16 q+4=0 \\
& q^{*}=\frac{4}{16}=\frac{1}{4} \\
& C=\frac{1}{4} \\
& D=\frac{3}{4} \\
& E\left(\Pi_{2}\right)=p q 25+p(1-q) 5+(1-p) q 10+(1-p)(1-q) 15 \\
& \frac{\partial E\left(\Pi_{2}\right)}{\partial q}=25 p-5 p+(1-p) 10-(1-p) 15=0 \\
& \frac{\partial E\left(\Pi_{2}\right)}{\partial q}=25 p-5=0 \\
& p^{*}=\frac{5}{25}=\frac{1}{5} \\
& A=\frac{1}{5} \\
& B=\frac{4}{5}
\end{aligned}
$$




\section{Notes}

1 Pindyck and Rubinfeld (2005) describe 'matching pennies' as, 'each player chooses heads or tails and the two players reveal their coins at the same time. If the coins match (i.e. both are heads or both are tails), Player $A$ wins and receives a dollar from Player B. If the coins do not match, Player B wins and receives a dollar from Player A.' A variant is for the winning player to receive the losing player's coin. The normal forms for the 'matching pennies' and 'rock-paper-scissors' games are given in the appendix.

2 These universities represent a (limited) cross-section of schools of higher education across the US. While this selection of universities is limited to the US, note that we have not found any textbook or article which presents the method we propose.

3 For example, player 2 's expected payoff from playing $C$ is $1 / 2^{*} 25+1 / 2^{*} 10=17.5$. Player 1 's expected payoff when player 2 plays $C$ is $1 / 2^{*} 4+1 / 2^{*} 16=10$. These are the expected payoffs listed in the cell resulting from player 2 playing $C$ and player 1 playing a mix of $1 / 2 A$ and $1 / 2 B$ in the bottom left corner of Figure 2 .

4 For a more thorough explanation about how to set up and derive the algebraic solutions, please see Hay et al.; Nicholson and Snyder; McGuigan et al.; Neilson and Winter; Pindyck and Rubinfeld; or Varian. We follow a fairly standard procedure when solving for the equilibrium mixed strategies: $P_{i k}$ indicates the probability that player $i$ plays strategy $k$. Note that a player's probability weights sum to one; i.e. $\mathrm{P}_{2 \mathrm{C}}+\mathrm{P}_{2 \mathrm{D}}=$ 1. Therefore $\mathrm{P}_{2 \mathrm{D}}=1-\mathrm{P}_{2 \mathrm{C}}$. Similarly, $\mathrm{P}_{1 \mathrm{~A}}+\mathrm{P}_{1 \mathrm{~B}}=1$ and therefore $\mathrm{P}_{1 \mathrm{~B}}=1-\mathrm{P}_{1 \mathrm{~A}}$.

5 Although not directly related to the topic, we also find that undergraduate students are interested to learn that games in the normal, or strategic, form generally have a finite and odd number of Nash equilibria (see Fudenberg and Tirole, 1996). We frequently have them solve a simple 'Meeting Game' which results in 3 Nash equilibria: 2 in pure strategies and 1 in mixed strategies. A nice, easy example is on page 247 in Mas-Colell et al. (1995).

\section{References}

Baldani, Jeffrey, James Bradfield and Robert W.Turner (2005) Mathematical Economics (2nd edition). Mason, $\mathrm{OH}$ :Thomson South-Western.

Bernheim, B. Douglas and Michael D. Whinston (2008) Microeconomics. New York, NY: McGraw-Hill Irwin.

Chiappori, Pierre-Andre, Steven Levitt and Timothy Groseclose (2002) 'Testing MixedStrategy Equilibria when Players are Heterogeneous:The Case of Penalty Kicks in Soccer', American Economic Review. Volume 92, September.

Fudenberg, Drew and Jean Tirole (1996) Game Theory. Cambridge and London:The MIT Press.

Hay, Frederick G., Christine Oughton and Andrew S. Skinner (1996) Intermediate Microeconomics: A Perspective on Price Theory. Manchester and New York: Manchester University Press.

Mas-Colell, Andreu, Michael D. Whinston and Jerry R. Green (1995) Microeconomic Theory. New York and Oxford: Oxford University Press.

McCain, Roger A. (2004) Game Theory: A Non-Technical Introduction to the Analysis of Strategy. Mason, $\mathrm{OH}$ :Thomas South-Western.

McGuigan, James R., R. Charles Moyer and Frederick H. de B. Harris (2005) Managerial Economics: Applications, Strategy, and Tactics. Mason, OH:Thomas South-Western. 
Neilson, William S. and Harold Winter (1998) Intermediate Microeconomics. Cincinnati, OH: South-Western College Publishing.

Nicholson, Walter and Christopher Snyder (2007) Intermediate Microeconomics and Its Application (10th edition). Mason, $\mathrm{OH}$ :Thomson South-Western.

Pindyck, Robert S. and Daniel L. Rubinfeld (2005) Microeconomics (6th edition). Upper Saddle River, NJ: Pearson Prentice Hall.

Varian, Hal R. (2006) Intermediate Microeconomics: A Modern Approach (7th edition). New York and London:W.W. Norton \& Company.

Walker, Mark and John Wooders (2001) 'Minimax Play at Wimbledon', American Economic Review. Volume 1, December.

\section{Contact details}

Evan Moore

Associate Professor and Head of the Department of Economics

Auburn University Montgomery

P.O. Box 244023

Montgomery, AL 36124-4023

USA

Email: emoore1@aum.edu 